\title{
Severity Detection of Red lesion in Diabetic Retinopathy using GUI
}

\author{
Nasiya S K ${ }^{1}$, Joby James ${ }^{2}$ \\ PG Student, Electronic and Communication, College of Engineering Kidangoor, Kottayam, APJ Abdul Kalam \\ Technological University ${ }^{1}$ \\ Asst. Prof, Dept of Electronic and Communication, College of Engineering Kidangoor, Kottayam²
}

\begin{abstract}
In modern world, Diabetic Retinopathy (DR) is the leading cause of blindness. DR is a common reason for blindness in the age group of 20-74 years. The main reason for red lesion in working age population is micro aneurysms (MA) and hemorrhages(HE).The first clinical observable lesion indication of diabetic retinopathy are red lesions. So that the detection of red lesions are the main problem for the prescreening system. Here the proposed work gives the automatic detection of red lesion using the new set of shape features, known as dynamic shape features (DSF). DSF parameter gives shape of flooding blood in images and by using this the severity can be analyzed. Another aim of this paper is developing a MATLAB based Graphical User Interface (GUI) which helps to find out the abnormality and severity by the ophthalmologist easily and accurately.
\end{abstract}

Keywords: Fundus image, MA, HE, Graphical User Interface, Support Vector Machine.

\section{INTRODUCTION}

Diabetic retinopathy is an eye disease due to diabetes. It is a complication of diabetic mellitus which may leads to blindness in work age population. Diabetic retinopathy are of two types they are:-Non Proliferative Diabetic Retinopathy(NPDR) and Proliferative Diabetic Retinopathy(PDR).In NPDR the damaged blood vessels leaks extra fluid and small amount of blood, but in PDR the blood vessels in the retina will close and it will prevent the blood flow in eye. It may leads to the formation of new blood vessels.

The main symptoms of DR are red lesions, which are mainly due to the swelling in the retinal blood vessels and the damages of retinal blood vessels through injury or diseases. These conditions can be termed as Micro aneurysms and Hemorrhages respectively. Here MA s are uniformly circular in shape and limited in size. It can be detected by using morphological operations like diameter closing and top hat transformation using linear structuring. To differentiate micro aneurysms from blood vessels are by the elongated structure of red lesions. These are the primary symptoms of DR. Retinal Hemorrhages are the severe level of DR. These different types of hemorrhages like dot, blot and flame .Dot HE and Ma are similar in shape so that they are difficult to differentiate from one another on the fundus images. In flame HE the blood leaks into the nerve fiber. Its shape is more elongated like nerve fibers. In blot hemorrhages more blood leaks into retina .Its shape is larger than dot HE. These are various in shape and having the irregular boarders.
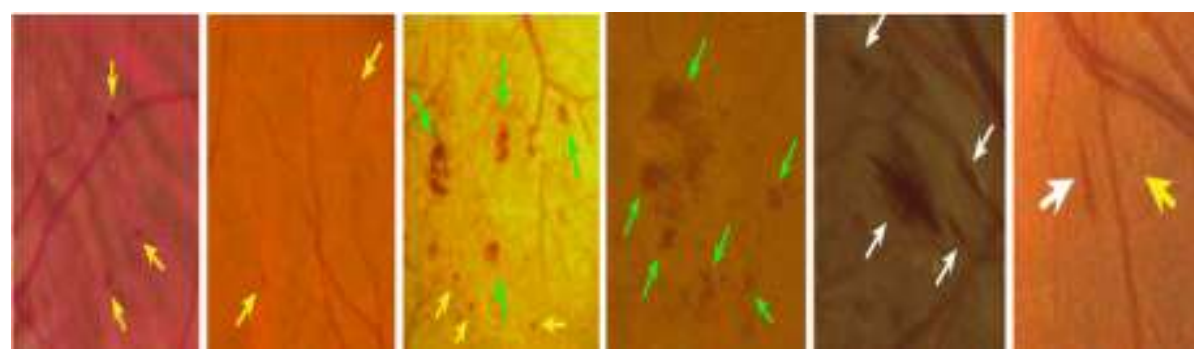

Figure 1: Different images of portions of red lesions. The yellow, green, white arrows represents Mas/dot HEs, blot HEs and flame HEs respectively [1].

In this paper MA and $\mathrm{HE}$ are detected by different steps such as image preprocessing, segmentation of preprocessing feature extraction, classification of lesion using SVM classifier and finally detecting the disorder. The final detection of disease is based on the DSF parameters like relative area, perimeter, eccentricity, solidity, circularity, rectangularity and elongation. An user interactive Graphical User Interface is implemented in this paper for finding the severity of the red lesions affected in the retina of patients. 


\section{PROPOSED METHOD}

The proposed system of this paper is differentiate the retinal disorders like HE and MA and its severity levels. The major steps for the detection carried out are image preprocessing, segmentation of lesions feature extraction ,classification of lesions using SVM classifiers and detecting the disorder.
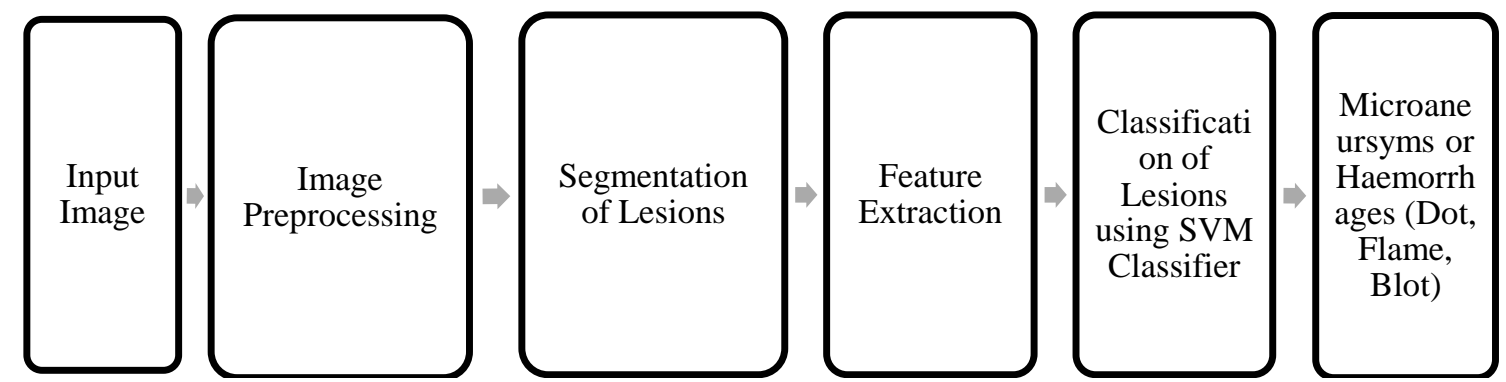

Block Diagram 3. 1:- Basis steps for red lesion detection

Image preprocessing techniques includes illumination equalization, denoising, RGB to gray conversion, adaptive thersholding and colour normalization. Segmentation of lesions includes two steps they are detection of blood vessels and detection of HE and MA .Detection of blood vessels helps the enhancement of the fundus image. Feature extraction is used for the classification of features obtained from the segmented retinal structures. Finally classifying the disorder based on the DSF parameters. The classification red lesion is used by support vector machine (SVM).SVM is based on structural risk minimization, which is statistical learning method. SVM is used to map the input image vector into a high dimensional feature space by using a non linear mapping kernel. The feature shape is given by Burges equation[2].

$$
\mathrm{f}(\mathrm{x})=\operatorname{sgm}\left[\sum_{\mathrm{i}=1}^{\mathrm{l}} \operatorname{yi\alpha ik}(\mathrm{xi}, \mathrm{x})+\mathrm{b}\right]
$$

here $\mathrm{k}$ is the kernel function, $\mathrm{b}$ is the bias vector , $\mathrm{ai}$ is the lagrange multiple and yi is the label..

In this paper SVM is used to classify the red lesion affected and not affected regions in the fundus images. For this purpose we can consider Niemeijer et al [3] features. The performance can be improved by considering the factors like angular second moment, contrast, correlation, sum of squares, inverse difference moment, sum average, sum variance, sum entropy, entropy, difference variance, different entropy and information measurements for correlation. All these features are obtained by co-occurrence matrix.

Severity of the red lesions are detected by the DSF parameters. Relative area is the one of the DSF parameters, in this the number of pixels is divided by the total number of pixels in the image. Elongation is obtained by $1-\mathrm{W} / \mathrm{L}$, where $\mathrm{W}$ and $\mathrm{L}$ are width and length of the images. Eccentricity is an another DSF parameter can be obtained from the width and length of the image by using the formulae $\sqrt{(\mathrm{L}-\mathrm{W}) / \mathrm{L}}$.

Finally classified disorder is detected on the GUI based system. GUI is an user interactive MATLAB tool so that even the patient can understand the severity of the disorder.

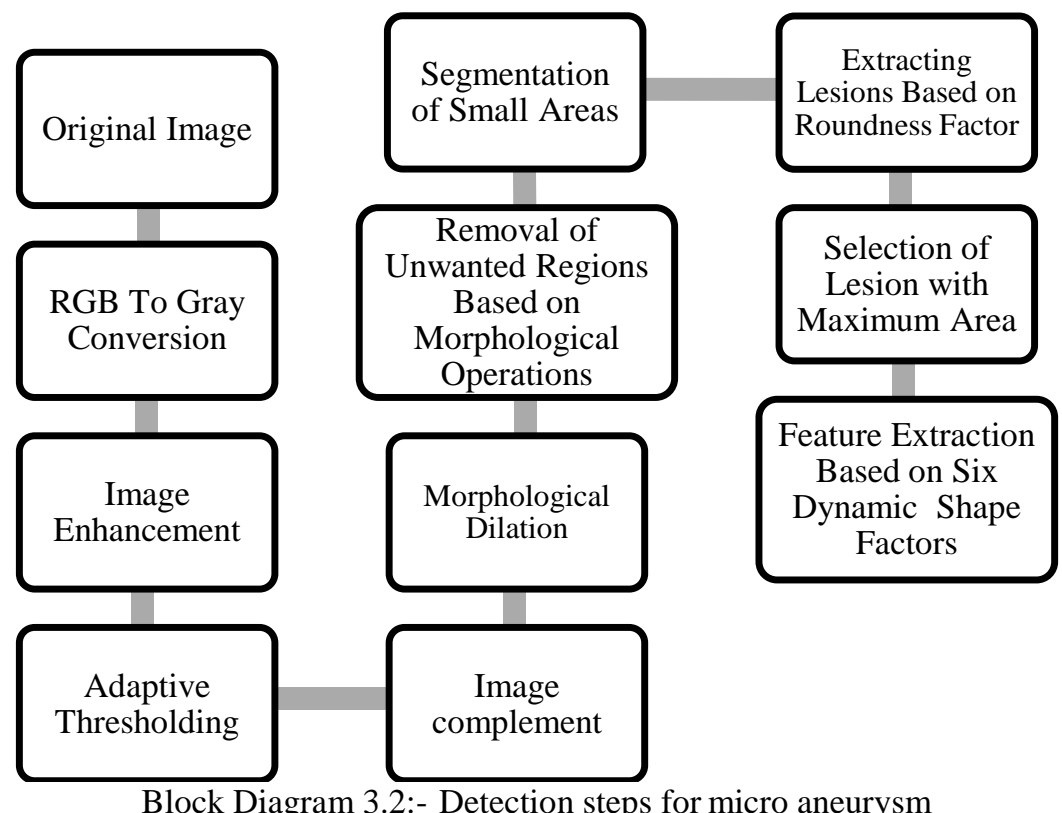


Vol. 6, Issue 4, April 2017

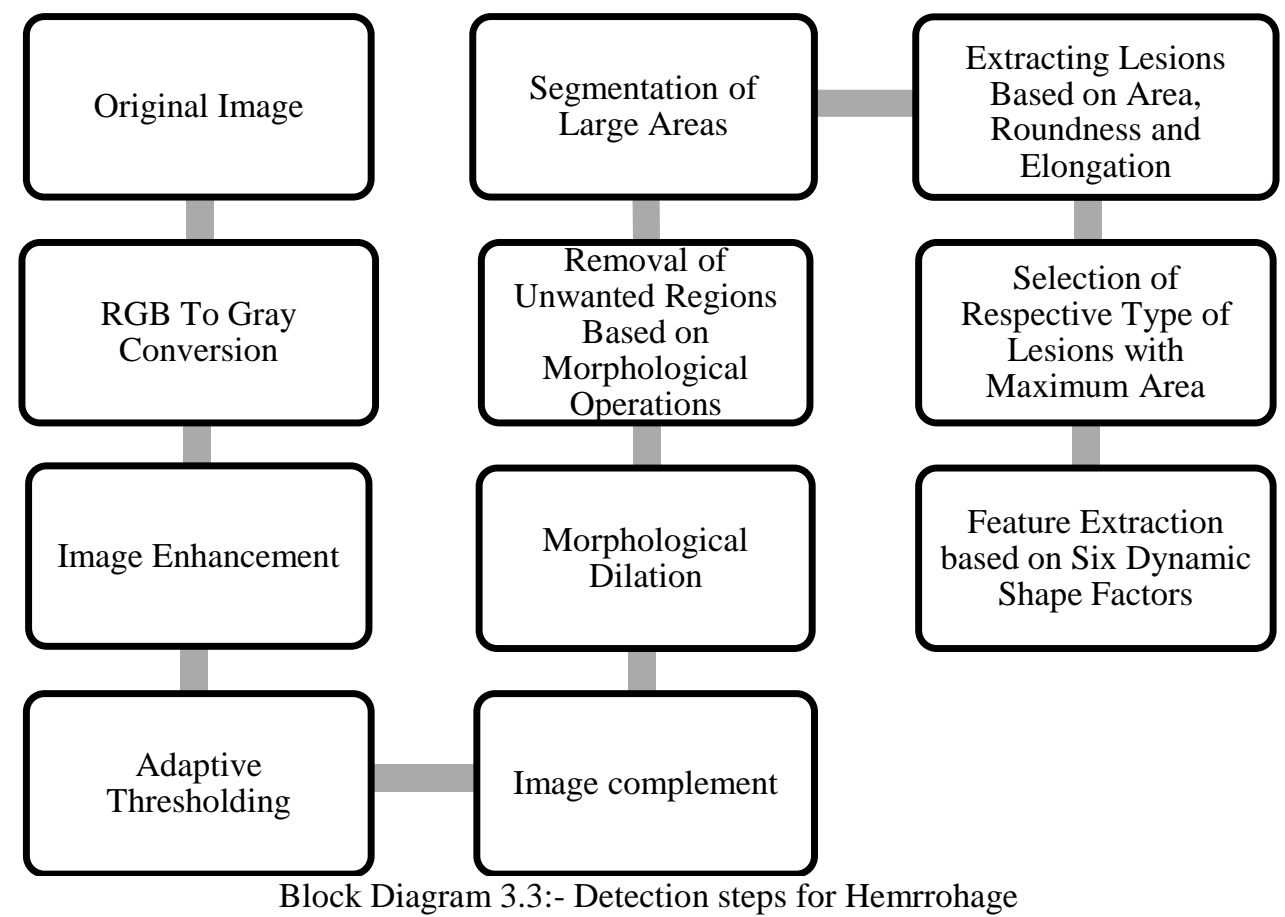

III.EXPERIMENTAL RESULTS

To analyze the effectiveness of the proposed method, algorithm is run on all the datasets and results for detection of red lesion and Blood Vessels were collected. Figure shows the resulting images of various stages in led lesion detection on a GUI screen and finally analyzing the severity of the fundus images by giving different threshold values for the various threshold values for different DSF parameters.

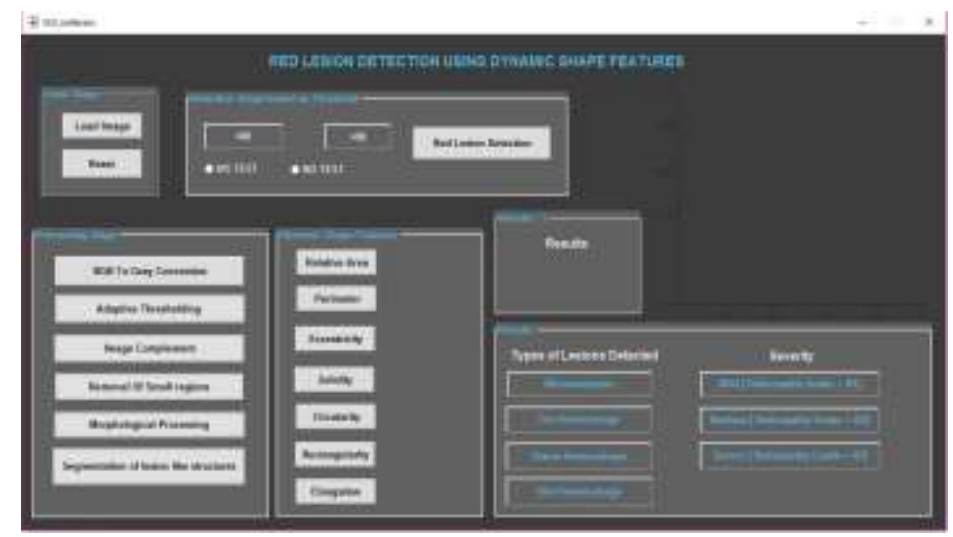

Fig 4.1: Generated GUI window

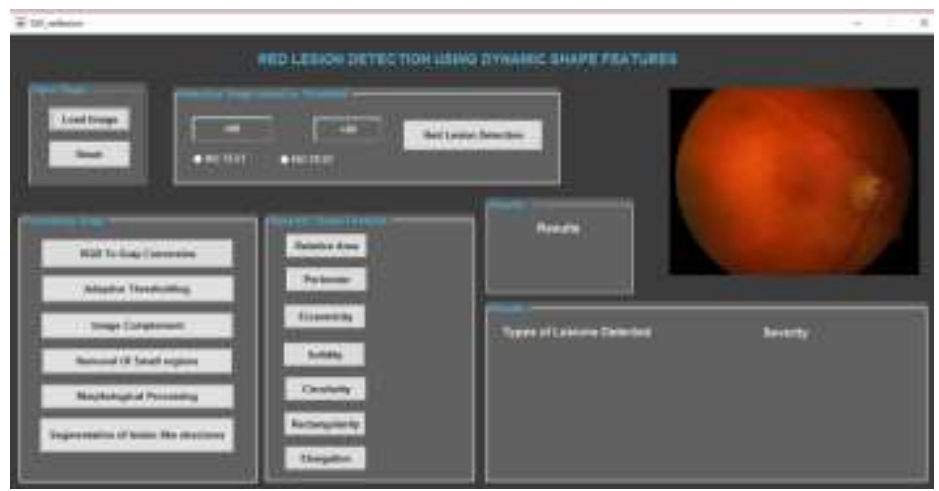

Fig 4.2: Loaded image 


\section{IJARCCE}

International Journal of Advanced Research in Computer and Communication Engineering ISO 3297:2007 Certified

Vol. 6, Issue 4, April 2017

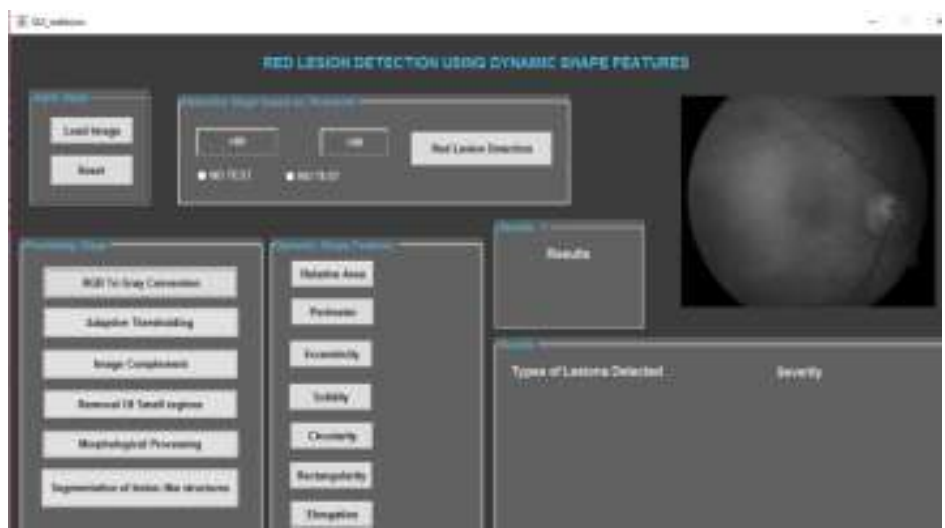

Fig 4.3 :RGB to Gray converted image

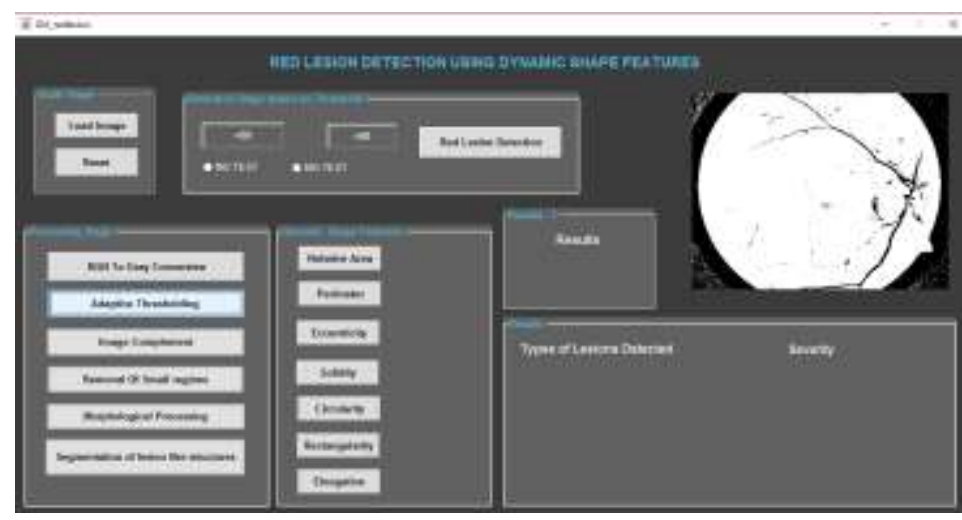

Fig 4.4: Adaptive Thersholding

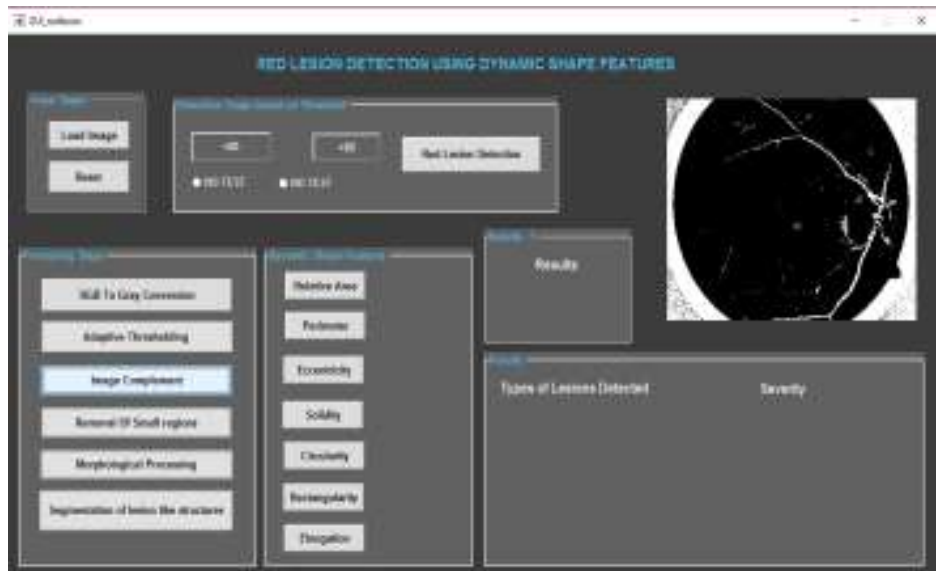

Fig 4.5 :Image Complement

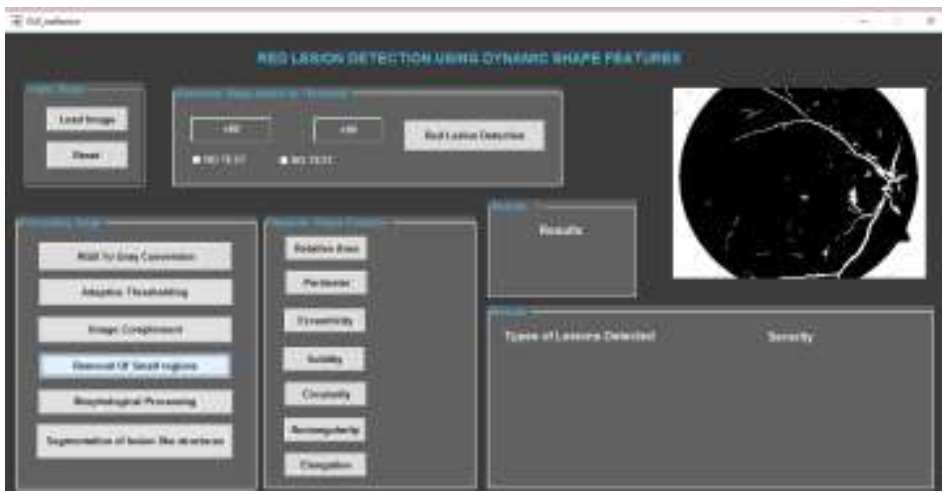

Fig 4.6: Removal of Small Regions 


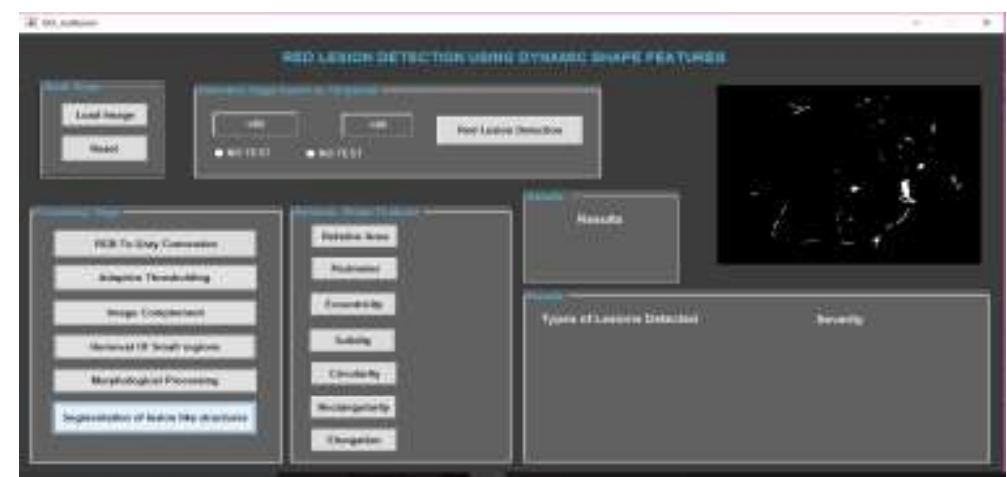

Fig 4.7: Segmentation of lesion like structures

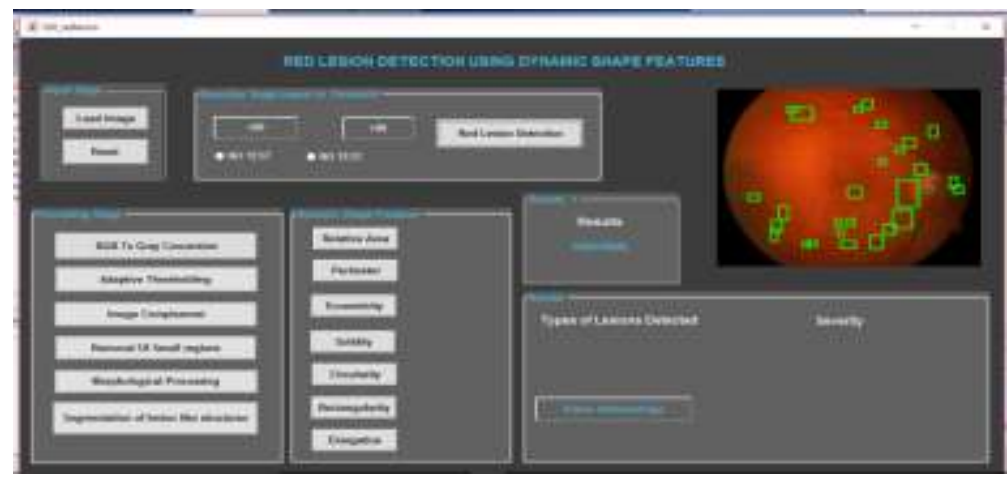

Fig 4.8: Detection of flame hemorrhage

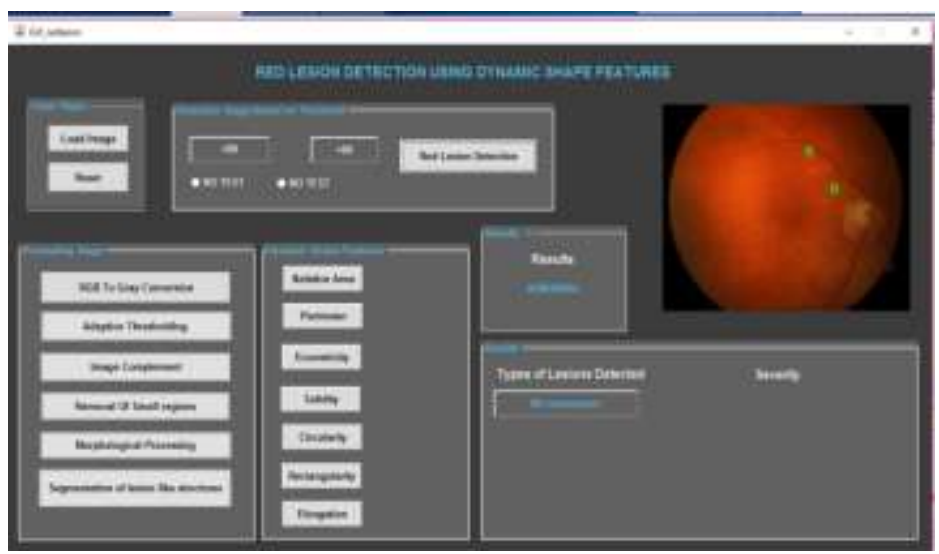

Fig 4.9: Detection of Micro aneurysm

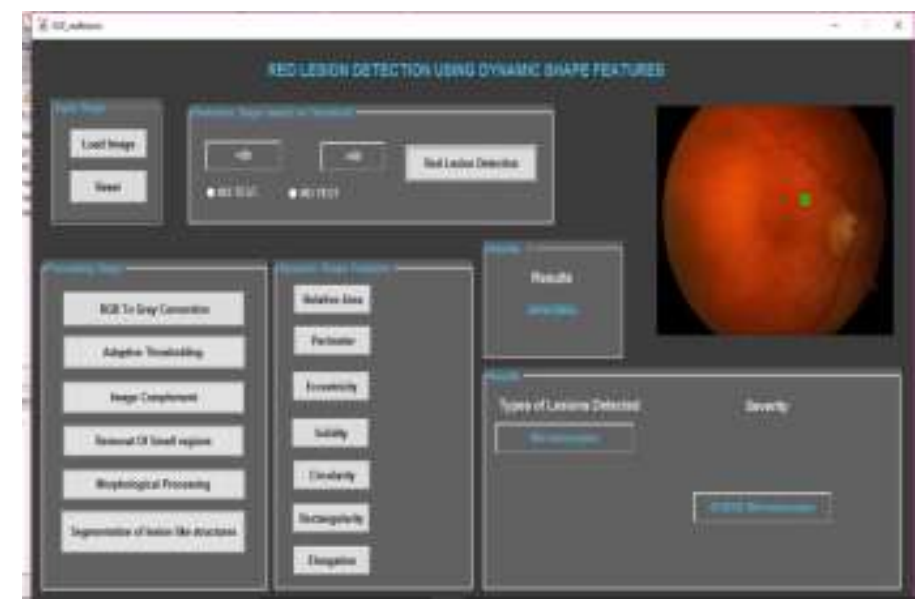

Fig 4.10: Detection of severe micro aneurysm 
Various diabetic retinopathy grading schemes are tabulated below:-

Table 4. 1 : Summary of the Diabetic Retinopathy Grading Scheme

\begin{tabular}{|l|l|}
\hline Grading & Description \\
\hline R0 (No visible retinopathy) & No visible diabetic retinopathy anywhere \\
\hline R1(Mild) & $\begin{array}{l}\text { At least one dot or flame hemorrhage, micro aneurysms, exudate or cotton wool } \\
\text { spot anywhere. }\end{array}$ \\
\hline R2(Observable background) & Four or more blot hemorrhage in one hemi field only. \\
\hline R3(Referable background) & $\begin{array}{l}\text { Any of the following features i) four or more blot hemorrhage in the inferior and } \\
\text { superior hemi fields ii)venous beading iii) intra retinal micro vascular anomalies. }\end{array}$ \\
\hline R4(Proliferative) & Any of the following features: i)active new vessels ii) vitreous hemorrhage \\
\hline R6(Inadequate) & Insufficient clarity or field of view \\
\hline
\end{tabular}

Different severity levels and its dilated ophthalmoscopy findings.

Table 4. 2: Severity level

\begin{tabular}{|l|l|}
\hline Disease severity level & Dilated ophthalmoscopy findings \\
\hline No apparent retinopathy & No abnormalities \\
\hline Mild NPDR & Micro aneurysms only \\
\hline Moderate NPDR & More than just micro aneurysms, but less than severe NPDR. \\
\hline Severe NPDR & No signs of PDR, with any of the following: \\
& Intra retinal hemorrhage \\
& Definite venous beading \\
& Prominent intra retinal micro vascular anomalies. \\
\hline PDR & Neovascularization, Vitreous or pre retinal hemorrhage. \\
\hline
\end{tabular}

\section{IV.CONCLUSION}

In this paper a novel red lesion detection based on the new set of dynamic shape features are carried out. The fundus image is processed in different steps for finding the severity of red lesion affected in diabetic patients. The Graphical Use Interface (GUI) is an user friendly tool used to identify severity levels of red lesion and it allows appropriate consistent referral to treatment center. The system can helps to reduce specialist's burden and examination time with the additional advantages of objectivity and reproducibility so that the implementation of this paper will be a succor to the patient as well to the ophthalmologist.

\section{REFERENCES}

[1]. Lama Seoud, Thomas Hurtut, Jihed Chelbi, Farida Cheriet and J.M. Pierre Langlois. "Red Lesion Detection using Dynamic Shape Features for Diabetic Retinopathy Screening “ IEEE TRANSACTIONS ON MEDICAL IMAGING, VOL. XX, NO. XX, XXX 201.

[2]. Burges CJC. A tutorial on support vector machines for pattern recognition. Data Min Knowl Discov. 1998;2:211-167. doi: 10.1023/A:1009715923555.

[3]. Niemeijer M, Ginneken B, Staal J, Suttorp-Schulten MSA, Abrmoff MD. Automatic detection of red lesions in digital color fundus photograph. IEEE Trans Med Imag. 2005;24(5):584592.

[4]. Haralick RM. Statistical and structural approaches to texture. Proc IEEE. 1979;67(5):786-804. doi: 10.1109/PROC.1979.11328.

[5] Alireza Osareh, Bita Shadgar and Richard Markham, 2009 A computational-intelligence-based approach for detection of exudates in diabetic retinopathy images. IEEE Transactions on Information Technology in Biomedicine, Vol. 13, no. 4, pp. 535-545.

[6] Akara Sopharak, Bunyarit Uyyanonvara, Sarah Barman, Thomas H. Williamson, 2008. Automatic detection of diabetic retinopathy exudates from non-dilated retinal images using mathematical morphology methods, Computerized Medical Imaging and Graphics 32, 720-727.

[7] Akara Sopharak, Bunyarit Uyyanonvara, Sarah Barman, 2009, "Automatic Exudate Detection for Diabetic Retinopathy Screening", ScienceAsia, $35,80-85$.

[8] Asha Gowda Karegowda, Asfiya Nasiha, M.A.Jayaram, 2011. Exudates Detection in Retinal Images using Back Propagation Neural Network, International Journal of Computer Applications (0975 - 8887), Volume 25, No.3.

[9] Doaa Youssef, N. Solouma, A. El-dib, M. Mabrouk, and A. B. Youssef, 2010. New Feature-Based Detection of Blood Vessels and Exudates in Color Fundus Images. IEEE International Conf. on Image Processing Theory Tools \& Applications. 294-299.

[10] Ege B.M., Hejlesen O.K., Larsen O.V., Moller K., Jennings B., Kerr D. and Cavan D.A., 2000. Screening for diabetic retinopathy using computer based image analysis and statistical classification. Computer Methods and Programs in Biomedicine, Vol. 62, no. 3, pp. $165-175$.

[11] Huan Wang, Wynne Hsu, Kheng Guan Goh and Mong Li Lee. 2000. An effective approach to detect lesions in retinal images. Proc. IEEE Conf. Comput. Vis. Pattern Recogn., Hilton Head Island, SC, Vol. 2, pp. 181-187.

[12] Huiqi Li, Opas Chutatape, 2004. Automated Feature Extraction in Color Retinal Images by a Model Based Approach, IEEE Transactions On Biomedical Engineering, Vol. 51, no. 2, pp. 246-254. 
[13] Lili Xu, Shuqian Luo, 2010. A novel method for blood vessel detection from retinal images, BioMedical Engineering OnLine , 9:14.

[14] Jagdish Nayak, P.Subbanna Bhat, Rajendra Acharya et al., 2008. Automated Identification of Diabetic Retinopathy Images using Digital Fundus Images. Journal of Medical Systems.32: 107-115.

[15] Neera Singh and Ramesh chandra tripathi, 2010. Automated Early Detection Of Diabetic Retinopathy Using Image Analysis Techniques. International Journal of Computer Applications. Vol.8,No.2, pp. 0975-8887.

[16] M. Niemeijer, B. V. Ginneken, S. R. Russell, M. Suttorp, and M. D. Abramoff, 2007. "Automated detection and differentiation of drusen, exudates and cotton-wool spots in digital color fundus photographs for diabetic retinopathy diagnosis," Invest. Ophthalmol. Vis. Sci., vol. 48, pp. 2260-2267.

[17] Rema M. and Pradeepa R., 2007. Diabetic retinopathy: an Indian perspective. Indian Journal of Medical Research, Vol. 125, no. 3, pp. 297.

[18] Saiprasad ravikumar et al., 2007. Automated Feature Extraction For Early Detection Of Diabetic Retinopathy In Funds Image. IEEE international conference on Image Processing, pp 139-142.

[19] C. I. Sánchez, R. Hornero, M. I. López, J. Poza, 2004, Retinal Image Analysis to Detect and Quantify Lesions Associated with Diabetic Retinopathy, Proceedings of the 26th Annual International Conference of the IEEE EMBS, September 1-5.

[20] Sinthanayothin C, Boyce JF, Williamson TH, Cook HL, Mensah E, Lal S, Usher D., 2002. Automated detection of diabetic retinopathy on digital fundus images. Diabetic Medicine.19(2), 105-12.

[21] Usher D, Dumskyj M, Himaga M, Williamson TH, Nussey S, Boyce J, 2004. Automated detection of diabetic retinopathy in digital retinal images: a tool for diabetic retinopathy screening. Diabet Med 21, 84-90.

[22] Prof B. Venkatalakshmi, V.Saravanan. GJenny Niveditha 2011, Graphical User Interface for Enhanced Retinal Image Analysis for Diagnosing Diabetic Retinopathy, 3 rd International Conference on Communication Software and Networks (ICCSN), 610-613.

[23] Thomas Walter, Jean-Claude Klein, Pascale Massin, and Ali Erginay, 2002. A contribution of image processing to the diagnosis of diabetic retinopathy-detection of exudates in color fundus images of the human retina. Medical Imaging, IEEE Transactions on, Vol. 21, no. 10, pp. 1236-1243.

[24] V.Vijaya Kumari, N.SuriyaNarayanan, 2010, Diabetic Retinopathy-Early Detection Using Image Processing Techniques, International Journal on Computer Science and Engineering, Vol. 02, No. 02, 357 